\title{
ON GENERATORS OF NEW METHODS OF THE PERTURBATION THEORY
}

\author{
E.A. GREBENIKOV \\ Institut of high-performance computing systems \\ $R A S$, Moscow
}

\begin{abstract}
In this article are discussed classical and modern interpretations of the perturbation theory methods.
\end{abstract}

\section{On the classical perturbation theory}

Let us consider an $n$-dimensional differential equation with small parameter $\mu$

$$
\frac{d z}{d t}=Z(z, t, \mu), \quad z(0)=z_{0}
$$

where the vector-function $Z(z, t, \mu)$ is determined and has properties guaranteeing the existence and uniqueness of the solutions of the Cauchy problem (1) in a $(n+1)$-dimensional domain $G_{(n+1)}=\{z \in G \times R \ni t\}$ of the Euclidean space. Our purpose is to construct this solution. Together with equation (1), we consider an equivalent one

$$
\frac{d z}{d t}=\bar{Z}(z, t, \mu)+Z(z, t, \mu)-\bar{Z}(z, t, \mu), \quad z(0)=z_{0},
$$

in which $\bar{Z}(z, t, \mu)$ is an arbitrary function. We write the linear equality

$$
z(t, \mu)=\bar{z}(t, \mu)+u(t, \mu)
$$

where $\bar{z}, u$ are some new unknown functions. The solution of a Cauchy problem can be found by solving the following two Cauchy problems:

$$
\frac{d \bar{z}}{d t}=\bar{Z}(\bar{z}, t, \mu), \quad \bar{z}(0)=\bar{z}_{0} \in G_{n}
$$




$$
\frac{d u}{d t}=Z(\bar{z}+u, t, \mu)-\bar{Z}(\bar{z}, t, \mu), \quad u(0)=z_{0}-\bar{z}_{0}
$$

where $\bar{z}_{0}$ is some new initial point. The equation (4) defines the choice of the initial approximation $\bar{z}(t, \mu)$ and equation (5) defines the total perturbation $u(t, \mu)$. From problem (5), one can see that perturbation $u(t, \mu)$ depends on the choice of function $\bar{Z}(\bar{z}, t, \mu)$, initial point $\bar{z}_{0}$ and, moreover, its finding is possible only after the solution of a equation (4). Thus, for a Cauchy problem (1), it is possible to construct a set of variants of the perturbation theory with parameters $\bar{Z}$ and $\bar{z}_{0}$. It is necessary that the solutions of equation (5) be "small" under the norm. We call $\bar{Z}(\bar{z}, t, \mu)$ and $\bar{z}_{0}$ the generators of the perturbation theory for problem (1) and equation (4) the generating equation for equation (1).

\section{New variants of the perturbation theory}

Now, we assume that the perturbation $u$ depends on $\bar{z}, t$ and $\mu$, that is, instead of (3) we have an equality

$$
z(t, \mu)=\bar{z}(t, \mu)+u(\bar{z}, t, \mu) .
$$

Therefore, instead of equations (4) and (5) we shall have equations (4) and (7):

$$
\frac{\partial u}{\partial t}+\left(\frac{\partial u}{\partial \bar{z}}, \bar{Z}(z, t, \mu)\right)=Z(\bar{z}+u, t, \mu)-\bar{Z}(\bar{z}, t, \mu), u(0)=z_{0}-\bar{z}_{0} .
$$

The perturbation theory based on equations (4) and (7) differs from the classical perturbation theory in an essential point: the determination of perturbation $u(\bar{z}, t, \mu)$ from the equation (7) does not require the preliminary solving of a generating equation (4).

So, let a problem of classical dynamics be described by a multifrequency system of $(m+n)$-order

$$
\left\{\begin{array}{l}
\frac{d x}{d t}=\mu X(x, y) \\
\frac{d y}{d t}=\omega(x)+\mu Y(x, y) .
\end{array}\right.
$$

where

$$
\begin{aligned}
& X(x, y)=\sum_{\|k\| \in I} X_{k}(x) e^{i(k, y)}, \quad Y(x, y)=\sum_{\|k\| \in I} Y_{k}(x) e^{i(k, y)}, \\
& i=\sqrt{-1}, \quad(k, y)=\sum_{s=1}^{n} k_{s} y_{s}, \quad\|k\|=\sum_{s=1}^{n}\left|k_{s}\right|, \quad I=\{0,1,2, \cdots\}
\end{aligned}
$$




$$
k_{s}=0, \pm 1, \cdots
$$

We choose, corresponding to (8), a generating system of the form

$$
\left\{\begin{array}{l}
\frac{d \bar{x}}{d t}=\mu \bar{X}(\bar{x}, \bar{y})+\sum_{k \geq 2} \mu^{k} A_{k}(\bar{x}, \bar{y}) \\
\frac{d \bar{y}}{d t}=\omega(x)+\mu \bar{Y}(\bar{x}, \bar{y})+\sum_{k \geq 2} \mu^{k} B_{k}(\bar{x}, \bar{y})
\end{array}\right.
$$

where $\bar{X}, \bar{Y}, A_{k}, B_{k}$ are arbitrary functions of their arguments. Let's look for the replacement of variable (6) as formal series

$$
x=\bar{x}+\sum_{k \geq 1} \mu^{k} u_{k}(\bar{x}, \bar{y}), \quad y=\bar{y}+\sum_{k \geq 1} \mu^{k} v_{k}(\bar{x}, \bar{y}),
$$

with unknown functions $u_{k}(\bar{x}, \bar{y}), v_{k}(\bar{x}, \bar{y})$. We have infinite system of linear partial differential equations of first order

$$
\left\{\begin{aligned}
&\left(\frac{\partial u_{1}}{\partial \bar{y}}, \omega(\bar{x})\right)=X(\bar{x}, \bar{y})-\bar{X}(\bar{x}, \bar{y}) \\
&\left(\frac{\partial v_{1}}{\partial \bar{y}}, \omega(\bar{x})\right)\left.=\left(\frac{\partial \omega}{\partial \bar{x}}, u_{1}\right)\right)+Y(\bar{x}, \bar{y})-\bar{Y}(\bar{x}, \bar{y}) \\
&\left(\frac{\partial u_{k}}{\partial \bar{y}}, \omega(\bar{x})\right)=F_{k}\left(\bar{x}, \bar{y}, u_{1}, v_{1}, \cdots, v_{k-1}, u_{k-1}, A_{2}, B_{2}, \cdots, A_{k}\right) \\
&\left(\frac{\partial v_{k}}{\partial \bar{y}}, \omega(\bar{x})\right)=\Psi_{k}\left(\bar{x}, \bar{y}, u_{1}, v_{1}, \cdots, v_{k-1}, u_{k}, A_{2}, B_{2}, \cdots, A_{k}, B_{k}\right), \\
& k=2,3, \cdots
\end{aligned}\right.
$$

The system (12) has a remarkable property: it is possible to integrate it in analytical way for any vector-index $k$ if, for functions $\bar{X}$ and $\bar{Y}$, are chosen some averages of the functions $X$ and $Y$. (9):

Really, let the generators $\bar{X}(\bar{x}, \bar{y}), \bar{Y}(\bar{x}, \bar{y})$ be the partial sums of series

$$
\bar{X}(\bar{x}, \bar{y})=\sum_{\|k\| \in I^{\prime}} X_{k}(\bar{x}) e^{i(k, \bar{y})}, \quad \bar{Y}(\bar{x}, \bar{y})=\sum_{\|k\| \in I^{\prime \prime}} Y_{k}(\bar{x}) e^{i(k, \bar{y})},
$$

where $I^{\prime} \in I$ and $I " \in I$ are "resonance sets". Then

$$
\left\{\begin{aligned}
X(\bar{x}, \bar{y})-\bar{X}(\bar{x}, \bar{y}) & =\sum_{\|k\| \in I \backslash I^{\prime}} X_{k}(\bar{x}) e^{i(k, \bar{y})}, \\
Y(\bar{x}, \bar{y})-\bar{Y}(\bar{x}, \bar{y}) & =\sum_{\|k\| \in I \backslash I^{\prime \prime}} Y_{k}(\bar{x}) e^{i(k, \bar{y})}
\end{aligned}\right.
$$


The sets $I \backslash I^{\prime}$ and $I \backslash I$ " are not "resonance sets". It is possible to find the exact solution of the first equations (12):

$$
\begin{gathered}
u_{1}(\bar{x}, \bar{y})=\sum_{\|k\| \in I \backslash I^{\prime}} \frac{X_{k}(\bar{x})}{i(k, \omega(\bar{x}))} e^{i(k, \bar{y})}+\varphi_{1}(\bar{x}) \\
v_{1}(\bar{x}, \bar{y})=\sum_{\|k\| \in I \backslash I^{\prime}} \frac{Y_{k}(\bar{x})}{i(k, \omega(\bar{x}))} e^{i(k, \bar{y})}+\left(\frac{\partial \omega(\bar{x})}{\partial x}, \sum_{\|k\| \in I \backslash I^{\prime}} \frac{X_{k}(\bar{x}) e^{i(k, \bar{y})}}{i^{2}(k, \omega(\bar{x}))^{2}}\right) \\
+\left(\left(\frac{\partial u_{1}}{\partial \bar{x}}, \varphi_{1}(\bar{x})\right), \bar{y}\right)+\psi_{1}(\bar{x}) .
\end{gathered}
$$

Here, $\psi_{1}, \varphi_{1}$ are arbitrary differentiable functions of their arguments $\bar{x}_{1}$, $\cdots, \bar{x}_{m}$.

Integration of equations (12) at $k=2,3, \cdots$ is without complicated difficulties. Rather important is the fact that, while determining functions $u_{2}$ and $v_{2}$, we can use functions $A_{2}, B_{2}, \psi_{1}, \varphi_{1}$.

The stated analytical algorithm means that we consequently construct the replacement of variables

$$
(x, y) \rightarrow\left(\bar{x}_{1}, \bar{y}_{1}\right) \rightarrow\left(\bar{x}_{2}, \bar{y}_{2}\right) \rightarrow \cdots \rightarrow\left(\bar{x}_{s}, \bar{y}_{s}\right) \rightarrow \cdots .
$$

Naturally, for the final construction of the solution of initial equations (8), one should solve the generating equation (10) with new initial conditions $\bar{x}(0), \bar{y}(0)$. In conclusion we want to note once again that, in formulas (11), the functions $u_{k}, v_{k}$ are found by analytical methods. The solution of the generating equation (10) can be found with the combination of numerical and analytical methods.

\section{References}

Grebenikov E., Ryabov J. 1983, Constructive methods in the analysis of nonlinear systems Moscow. Ed.MIR, 324 p. 